\section{Tendências no uso de serviços de saúde médicos e odontológicos e a relação com nível educacional e posse de plano privado de saúde no Brasil, 1998-2013}

\author{
Trends in the use of medical and dental services \\ and associations with educational level and \\ private health plan coverage in Brazil, 1998-2013
}

Tendencias en el uso de servicios de salud médicos y odontológicos, y su relación con el nivel educacional y la adquisición de un plan privado de salud en Brasil, 1998-2013

\section{Resumo}

O mix público-privado do sistema de saúde brasileiro favorece cobertura duplicada aos serviços de saúde aos indivíduos que possuem plano privado de saúde e pode aumentar as iniquidades no uso dos serviços. O objetivo deste estudo é descrever as tendências no uso dos serviços de saúde médicos e odontológicos e a relação com nível educacional e posse de plano privado de saúde. Os dados foram obtidos de inquéritos domiciliares nacionais com amostras representativas dos anos de 1998, 2003, 2008 e 2013. Foram descritas as tendências no uso de serviços de saúde por adultos ajustadas por posse de plano privado de saúde, nível de educação, sexo e idade. Há tendência de aumento no uso dos serviços de saúde em adultos sem plano privado e, entre adultos com plano privado, a tendência no uso variou de forma não linear. O serviço médico apresentou alternância no uso a longo dos anos e o serviço odontológico apresentou tendência de declínio após o ano de 2003. Acompanhar as tendências na posse de planos privados de saúde e no uso dos serviços de saúde é necessário para auxiliar o Estado na regulação dos planos privados e evitar o aumento das iniquidades no acesso e uso dos serviços de saúde entre os cidadãos.

Serviços de Saúde; Cobertura Universal; Seguro Saúde
Luciane Maria Pilotto 1

Roger Keller Celeste 2

doi: 10.1590/0102-311X00052017

\author{
Correspondência \\ L. M Pilotto \\ Universidade do Vale do Taquari UNIVATES. \\ Av. Avelini Talini 171, Lajeado, RS 95900-000, Brasil. \\ lutipilotto@yahoo.com.br \\ 1 Universidade do Vale do Taquari UNIVATES, Lajeado, Brasil. \\ 2 Universidade Federal do Rio Grande do Sul, Porto Alegre, \\ Brasil.
}




\section{Introdução}

Em 2005, os Estados Membros da Organização Mundial da Saúde comprometeram-se com a expansão de sistemas de saúde com cobertura universal, que ofereçam às pessoas os serviços de saúde de que necessitam ${ }^{1}$ a fim de proteger dos riscos financeiros catastróficos decorrentes do pagamento direto dos cuidados em saúde 2 . O Brasil possui um sistema público de saúde com cobertura universal (Sistema Único de Saúde - SUS), defendendo a saúde como um direito de todos e dever do Estado. Apesar disso, o sistema de saúde brasileiro é formado por um mix público e privado, de prestadores e compradores de serviços que se complementam e competem entre si, possuindo três subsetores distintos - público, privado e da saúde suplementar ${ }^{3}$. De acordo com a classificação da Organização para a Cooperação e Desenvolvimento Econômico (OECD) 4, os planos privados de saúde exercem um papel duplicado dentro do sistema de saúde brasileiro pois cobrem serviços de saúde já ofertados pelo sistema público. Nos últimos 15 anos, houve um aumento progressivo na posse de plano privado entre os brasileiros, passando de um quarto da população 5. Assim, essa parcela da população pode acessar o sistema de saúde de forma duplicada, tanto pelo uso do serviço público quanto pelo uso por meio do plano privado. A existência desse sistema de saúde misto resulta em desigualdades e iniquidades no acesso aos serviços de saúde 6 , permanecendo grandes disparidades socioeconômicas no uso de serviços médicos 7 e odontológicos 8 pela população. A posse de plano privado de saúde favorece a iniquidade em direção dos mais ricos 9 .

Estudos transversais mostram que pessoas com posse de plano privado de saúde utilizaram mais os serviços de saúde em relação àquelas pessoas que não possuem cobertura 10,11,12 e relataram melhores condições de saúde em relação aos indivíduos sem plano ${ }^{13}$. Estudos de tendência das condições de saúde 14 e da equidade horizontal no uso de serviços 9 indicam que houve um aumento no uso de serviços de saúde no Brasil de 1998 para 2008, provavelmente pela expansão da Estratégia Saúde da Família (ESF). Estudo de tendência sobre as desigualdades sociais na utilização dos serviços médicos e odontológicos mostrou desigualdades persistentes com maior proporção de uso entre indivíduos de maior renda 7,8. Não foram encontrados estudos de tendências no uso de serviços de saúde comparando médico com odontológico no Brasil. É provável que tenha ocorrido um aumento no uso dos serviços devido à expansão da cobertura pela ESF, mas o aumento no percentual de pessoas com plano privado de saúde também pode ter contribuído para esse fato. O uso de serviço médico e odontológico ocorre de maneira diferente, o aumento da idade faz com que ocorra aumento no uso do serviço médico e redução no uso do serviço odontológico. Além disso, estudos mostraram que cerca de 50\% das consultas odontológicas foram realizadas com pagamento direto, e $60 \%$ das consultas médicas foram feitas no SUS 10,15. Considerando o mix público-privado do sistema de saúde brasileiro e as iniquidades no uso de serviços entre os cidadãos com acesso duplicado, o objetivo deste estudo é descrever as tendências no uso dos serviços de saúde médico e odontológico em relação à posse de plano privado de saúde e nível educacional.

\section{Métodos}

Este é um estudo de análise de tendência temporal que utilizou dados da Pesquisa Nacional por Amostra de Domicílios (PNAD) de 1998, 2003 e 2008 e dados da Pesquisa Nacional de Saúde (PNS) realizada em 2013. Essas pesquisas são realizadas pelo Instituto Brasileiro de Geografia e Estatística (IBGE) em colaboração com o Ministério da Saúde. A proposta da PNAD é produzir informações gerais sobre o desenvolvimento social e econômico do país, e há um suplemento específico para saúde 16,17,18. A PNS tem por objetivo coletar informações para acompanhar o desempenho dos serviços de saúde e para a vigilância dos fatores de risco comportamentais 19 . Existem algumas diferenças em relação às variáveis coletadas nas PNADs realizadas em 1998, 2003 e 2008 e na PNS em 2013, algumas foram excluídas e outras incluídas ou modificadas. Apesar dessas pequenas alterações, é possível manter uma série histórica que permite comparações entre os anos 20,21. Ambos os estudos usam amostra probabilística complexa de três estágios e são representativos da população. Em 1998, 2003, 2008 e 2013 participaram da pesquisa 344.975, 384.834, 391.868 e 205.546 pessoas, respectivamente. Para este 
trabalho, foram selecionados os adultos, com idade entre 18 e 59 anos, totalizando 760.767 pessoas. Mais detalhes podem ser encontrados na Figura 1.

Todas as variáveis deste estudo foram retiradas das pesquisas nacionais coletadas com a mesma questão ou questão equivalente repetida em todos os anos. A variável relacionada ao uso de serviços médicos (usou/não usou) nos anos de 1998, 2003 e 2008 foi coletada pela questão "Nos últimos 12 meses ______ consultou médico?". A categoria "usou” incluiu todas as pessoas que responderam "sim", e os que responderam "não" foram alocados na categoria "não usou”. No ano de 2013, a questão utilizada foi "Quando _______ consultou médico pela última vez?”. Os indivíduos que responderam "Nos 12 últimos meses" foram alocados para a categoria "usou", e os que responderam "De 1 a menos de 2 anos", "De 2 anos a menos de 3 anos", "3 anos ou mais” ou "Nunca foi ao médico" foram colocados na categoria "não usou”. Para o uso do serviço odontológico, foi utilizada a questão "Quando foi

\section{Figura 1}

Quadro metodológico e questões apresentadas nos inquéritos.

\begin{tabular}{|c|c|c|c|c|}
\hline & PNAD 1998 & PNAD 2003 & PNAD 2008 & PNS 2013 \\
\hline Abrangência & $\begin{array}{c}\text { Todas as áreas } \\
\text { geográficas, com } \\
\text { exceção das áreas rurais } \\
\text { de Acre, Rondônia, Pará, } \\
\text { Amazonas, Roraima } \\
\text { e Amapá }\end{array}$ & $\begin{array}{c}\text { Todas as áreas } \\
\text { geográficas, com } \\
\text { exceção das áreas rurais } \\
\text { de Acre, Rondônia, Pará, } \\
\text { Amazonas, Roraima } \\
\text { e Amapá }\end{array}$ & $\begin{array}{c}\text { Todas as áreas } \\
\text { geográficas }\end{array}$ & Todas as áreas geográficas \\
\hline Plano amostral & $\begin{array}{l}\text { Três estágios de seleção: } \\
\text { unidades primárias - } \\
\text { municípios; unidades } \\
\text { secundárias - setores } \\
\text { censitários; e unidades } \\
\text { terciárias - unidades } \\
\text { domiciliares }\end{array}$ & $\begin{array}{l}\text { Três estágios de seleção: } \\
\text { unidades primárias - } \\
\text { municípios; unidades } \\
\text { secundárias - setores } \\
\text { censitários; e unidades } \\
\text { terciárias - unidades } \\
\text { domiciliares }\end{array}$ & $\begin{array}{l}\text { Três estágios de seleção: } \\
\text { unidades primárias - } \\
\text { municípios; unidades } \\
\text { secundárias - setores } \\
\text { censitários; e unidades } \\
\text { terciárias - unidades } \\
\text { domiciliares }\end{array}$ & $\begin{array}{c}\text { Três estágios de seleção: } \\
\text { unidades primárias - setores } \\
\text { censitários; unidades } \\
\text { secundárias - domicílios; } \\
\text { e unidades terciárias - } \\
\text { moradores de } 18 \text { anos } \\
\text { ou mais }\end{array}$ \\
\hline Amostra & $\begin{array}{l}344.975 \text { pessoas e } \\
112.434 \text { domicílios }\end{array}$ & $\begin{array}{l}\text { 383.834 pessoas e } \\
\text { 133.255 domicílios }\end{array}$ & $\begin{array}{l}391.868 \text { pessoas e } \\
150.591 \text { domicílios }\end{array}$ & $\begin{array}{c}205.546 \text { pessoas e } 81.357 \\
\text { domicílios }\end{array}$ \\
\hline Consulta médica & $\begin{array}{l}\text { v1347 Nos últimos } 12 \\
\text { meses__consultou } \\
\text { médico? }\end{array}$ & $\begin{array}{l}\text { v1347 Nos últimos } 12 \\
\text { meses__consultou } \\
\text { médico? }\end{array}$ & $\begin{array}{l}\text { v1347 Nos } 12 \text { últimos } \\
\text { meses__ consultou } \\
\text { médico? }\end{array}$ & $\begin{array}{l}\text { J011 Quando __ consultou } \\
\text { um médico pela } \\
\text { última vez? }\end{array}$ \\
\hline Consulta odontológica & $\begin{array}{l}\text { v1349 Quando __ foi ao } \\
\text { dentista pela última vez? }\end{array}$ & $\begin{array}{l}\text { v1349 Quando __ foi ao } \\
\text { dentista pela última vez? }\end{array}$ & $\begin{array}{l}\text { v3349 Quando __ foi ao } \\
\text { dentista pela última vez? }\end{array}$ & $\begin{array}{l}\text { J013 Quando __ consultou } \\
\text { um dentista pela última vez? }\end{array}$ \\
\hline Plano privado de saúde & $\begin{array}{l}\text { V1321__ tem direito } \\
\text { a algum plano de } \\
\text { saúde (médico ou } \\
\text { odontológico), particular, } \\
\text { de empresa ou órgão } \\
\text { público? }\end{array}$ & $\begin{array}{l}\text { V1321__ tem direito } \\
\text { a algum plano de } \\
\text { saúde (médico ou } \\
\text { odontológico), particular, } \\
\text { de empresa ou órgão } \\
\text { público? }\end{array}$ & $\begin{array}{l}\text { V1321 _ tem direito a } \\
\text { algum plano de saúde, } \\
\text { médico ou odontológico, } \\
\text { particular, de empresa } \\
\text { ou órgão público? }\end{array}$ & $\begin{array}{l}1001 \text { __ tem algum plano } \\
\text { de saúde (médico ou } \\
\text { odontológico), particular, de } \\
\text { empresa ou órgão público? }\end{array}$ \\
\hline $\begin{array}{l}\text { Plano privado de } \\
\text { saúde exclusivamente } \\
\text { odontológico }\end{array}$ & $\begin{array}{l}\text { v1344 __ tem algum } \\
\text { outro plano de saúde } \\
\text { apenas para assistência } \\
\text { odontológica? }\end{array}$ & $\begin{array}{l}\text { v1344 _ _ tem algum } \\
\text { outro plano de saúde } \\
\text { apenas para assistência } \\
\text { odontológica? }\end{array}$ & $\begin{array}{l}\text { v1344 __ tem algum } \\
\text { outro plano de saúde } \\
\text { apenas para assistência } \\
\text { odontológica? }\end{array}$ & $\begin{array}{l}1003 \text { __ tem algum plano } \\
\text { de saúde apenas para } \\
\text { assistência odontológica? }\end{array}$ \\
\hline
\end{tabular}

PNAD: Pesquisa Nacional por Amostra de Domicílios; PNS: Pesquisa Nacional de Saúde. 
ao dentista pela última vez?”. Os indivíduos que responderam ter ido ao dentista em menos de um ano foram classificados como "usou", os demais foram incluídos na categoria "não usou".

A variável referente ao tipo de plano privado de saúde resultou da combinação das questões: (1) “______ tem direito a algum plano de saúde, médico ou odontológico, particular, de empresa ou órgão público?” e (2) “_______ tem algum plano de saúde apenas para assistência odontológica?”. Para avaliar o serviço médico, essa variável foi classificada como: (a) plano médico - incluindo todas as pessoas que responderam possuir plano privado que não era exclusivamente odontológico e (b) sem plano médico - demais pessoas. Para analisar o uso de serviços odontológicos, a variável tipo de plano de saúde foi categorizada em: (a) plano odontológico - para indivíduos que responderam ter direito a plano exclusivamente odontológico e (b) sem plano odontológico - demais indivíduos (incluindo aqueles com plano médico).

Demais variáveis utilizadas no estudo foram sexo (masculino e feminino), grupo etário (18-19, 20-29, 30-39, 40-49, 50-59 anos) e nível educacional (Ensino Fundamental inicial incompleto ou menos, Ensino Fundamental inicial, Ensino Fundamental final, Ensino Médio ou mais). A variável sobre nível educacional foi criada com uma combinação de perguntas considerando se sabe ler e escrever, se frequenta escola, o curso que frequenta ou frequentou, a última série/curso que frequentou e se concluiu curso/série com aprovação.

As tendências no percentual de pessoas que utilizaram os serviços de saúde foram apresentadas em tabelas específicas para cada serviço de saúde, médico e odontológico, de acordo com o tipo de plano, sexo, grupo etário e nível educacional. O teste de significância para variáveis categóricas foi o qui-quadrado de Pearson com linearização de Taylor. Análises ajustadas foram realizadas por regressão logística. A tendência no uso dos serviços foi testada por meio de um termo de interação entre o ano do inquérito e as demais variáveis. Em relação ao nível educacional e ao grupo etário, o ano entrou como variável contínua e, para posse de plano, como uma dummy. As análises estatísticas foram realizadas utilizando o software Stata, versão 13.1 (StataCorp LP, College Station, Estados Unidos), empregando o comando de survey (svy) para incorporar os pesos amostrais fornecidos com os bancos.

\section{Resultados}

As amostras foram compostas por 188.766, 219.828, 230.408 e 121.765 adultos em 1998, 2003, 2008 e 2013, respectivamente, totalizando 760.767 indivíduos. A amostra final para a análise de tendência incluiu 754.014 pessoas para o serviço médico e 753.977 adultos no serviço odontológico, apresentando $0,9 \%$ de perdas. O percentual de mulheres foi maior em todos os anos; a proporção de indivíduos com menor escolaridade diminuiu no período em mais de $50 \%$, ao passo que o percentual de maior nível educacional aumentou em maior proporção. A Tabela 1 mostra que a posse de plano privado médico diminuiu progressivamente de 1998 para 2013, variando de 24,9\% a 22,2\% da população, porém, o uso dos serviços médicos aumentou progressivamente passando de $55,2 \%$ para $71,3 \%$. Já entre os planos privados exclusivamente odontológicos, houve um aumento nesse mesmo período, passando de $1 \%$ para $6,3 \%$, e o percentual de adultos que consultou dentista no último ano passou de $35,2 \%$ para $47 \%$.

A Tabela 2 apresenta os percentuais de consultas médicas e odontológicas no último ano em cada ano analisado. O uso dos serviços médico e odontológico foi maior entre os adultos com posse de plano privado, dentre as mulheres e para pessoas com maior nível de escolaridade em todos os anos. No entanto, houve tendência de aumento do uso dos serviços ao longo dos anos por todos os indivíduos, independente de posse de plano, sexo, escolaridade ou faixa etária. A idade apresentou tendências inversas comparando o uso do serviço médico com o odontológico. Diferentemente do uso de serviços médicos, as pessoas com maior idade tendem a utilizar menos os serviços odontológicos. As diferenças no uso de serviços odontológicos entre os grupos etários têm diminuído, mas as diferenças no uso de serviços médicos têm aumentado. De 1998 para 2013, houve um aumento no uso de serviços médicos e odontológicos por indivíduos com e sem plano privado, por homens e mulheres, independente do nível de instrução e faixa etária.

A Tabela 3 mostra que houve um aumento no uso dos serviços médicos e odontológicos entre os indivíduos sem plano em relação ao ano de 1998, independente das mudanças por covariantes socio- 


\section{Tabela 1}

Frequência absoluta e frequência relativa utilizando pesos amostrais das variáveis do estudo de acordo com o ano. Pesquisa Nacional por Amostra de Domicílios (PNAD 1998, 2003 e 2008) e Pesquisa Nacional de Saúde (PNS 2013), Brasil.

\begin{tabular}{|c|c|c|c|c|c|c|c|c|}
\hline \multirow[t]{2}{*}{ Amostra } & \multicolumn{2}{|c|}{$1998[N=188.766]$} & \multicolumn{2}{|c|}{$2003[N=219.828]$} & \multicolumn{2}{|c|}{$2008[N=230.408]$} & \multicolumn{2}{|c|}{$2013[N=121.765$} \\
\hline & $\mathbf{n}$ & $\%$ & $\mathbf{n}$ & $\%$ & $\mathbf{n}$ & $\%$ & $\mathbf{n}$ & $\%$ \\
\hline \multicolumn{9}{|l|}{ Plano privado exclusivamente odontológico } \\
\hline Com plano & 2.122 & 1,0 & 4.145 & 1,9 & 9.626 & 4,1 & 6.919 & 6,3 \\
\hline Sem plano & 184.887 & 99,0 & 212.975 & 98,1 & 220.782 & 95,9 & 114.848 & 93,7 \\
\hline \multicolumn{9}{|l|}{ Plano privado de assistência médica } \\
\hline Com plano & 46.251 & 24,9 & 49.327 & 23,7 & 51.969 & 23,7 & 25.082 & 22,2 \\
\hline Sem plano & 140.758 & 75,1 & 167.793 & 76,3 & 178.439 & 76,3 & 96.683 & 77,8 \\
\hline \multicolumn{9}{|l|}{ Uso dos serviços médicos } \\
\hline Usou & 106.260 & 55,2 & 137.228 & 62,3 & 155.680 & 67,7 & 81.463 & 71,3 \\
\hline Não usou & 82.466 & 44,8 & 82.578 & 37,7 & 74.728 & 32,3 & 40.302 & 28,7 \\
\hline \multicolumn{9}{|l|}{ Uso dos serviços odontológicos } \\
\hline Usou & 68.308 & 35,2 & 90.051 & 41,0 & 98.107 & 42,7 & 55.332 & 47,0 \\
\hline Não usou & 120.399 & 64,9 & 129.747 & 59,0 & 132.301 & 57,4 & 66.433 & 53,0 \\
\hline \multicolumn{9}{|l|}{ Sexo } \\
\hline Masculino & 91.020 & 48,5 & 106.014 & 48,3 & 111.181 & 48,4 & 58.279 & 45,7 \\
\hline Feminino & 97.746 & 51,5 & 113.814 & 51,7 & 119.227 & 51,6 & 63.486 & 54,3 \\
\hline \multicolumn{9}{|l|}{ Nível de instrução } \\
\hline Ensino Fundamental inicial incompleto ou menos & 52.834 & 28,9 & 49.570 & 22,8 & 40.994 & 17,8 & 21.002 & 16,8 \\
\hline Ensino Fundamental inicial & 59.206 & 31,6 & 60.278 & 27,8 & 52.938 & 23,1 & 19.793 & 17,3 \\
\hline Ensino Fundamental final & 28.214 & 14,7 & 36.277 & 16,2 & 39.166 & 16,7 & 18.565 & 15,5 \\
\hline Ensino Médio ou mais & 47.526 & 24,8 & 72.341 & 33,2 & 97.310 & 42,4 & 62.405 & 50,4 \\
\hline \multicolumn{9}{|l|}{ Faixa etária (anos) } \\
\hline $18-19$ & 14.254 & 7,5 & 15.170 & 6,8 & 13.861 & 6,0 & 7.514 & 5,1 \\
\hline $20-29$ & 58.227 & 30,2 & 37.477 & 30,9 & 34.776 & 29,4 & 16.940 & 25,0 \\
\hline $30-39$ & 51.519 & 27,1 & 57.370 & 25,9 & 58.415 & 25,2 & 32.381 & 27,9 \\
\hline $40-49$ & 39.442 & 21,3 & 46.778 & 21,7 & 51.825 & 22,7 & 27.181 & 22,9 \\
\hline $50-59$ & 25.324 & 13,9 & 31.086 & 14,7 & 37.461 & 16,7 & 21.339 & 19,1 \\
\hline
\end{tabular}

demográficas. Dentre os indivíduos que têm plano privado de saúde, também foi observado aumento ao longo do tempo e, embora indivíduos com plano apresentem maior uso em todos os anos, a diferença flutuou. O uso do serviço odontológico apresentou um aumento de 1998 para os demais anos, sendo esse maior de 1998 para 2003 e apresentou tendência de declínio de 2003 para 2013, apesar do percentual de adultos com plano exclusivamente odontológico ter aumentado em maior proporção nos últimos dois anos. Houve tendência de aumento do uso dos serviços médicos e odontológicos conforme aumentava a escolaridade; porém, o efeito adicional da escolaridade, com o passar dos anos, mostrou redução nas desigualdades educacionais em relação ao serviço odontológico e aumento das desigualdades educacionais em relação ao serviço médico.

\section{Discussão}

Este estudo mostrou que houve tendência de aumento no uso dos serviços de saúde médicos e odontológicos dentre indivíduos com e sem plano privado de saúde e o percentual de uso de serviços no último ano foi maior para pessoas com posse de planos em todos os anos. No entanto, as tendências nas diferenças entre usuários com e sem plano variou de forma não linear. Os serviços médicos apresentaram alternância ao longo do período analisado e os serviços odontológicos, apesar de evidencia- 


\section{Tabela 2}

Percentual de uso de serviços médico e odontológico no último ano entre adultos de acordo com a posse de plano privado de saúde, sexo, nível de instrução e faixa etária. Pesquisa Nacional por Amostra de Domicílios (PNAD 1998, 2003 e 2008) e Pesquisa Nacional de Saúde (PNS 2013), Brasil *.

\begin{tabular}{|c|c|c|c|c|c|c|c|c|c|c|}
\hline \multirow[t]{2}{*}{ Amostra } & \multicolumn{5}{|c|}{ Consulta médica } & \multicolumn{5}{|c|}{ Consulta odontológica } \\
\hline & 1998 & 2003 & 2008 & 2013 & Valor de $p$ ** & 1998 & 2003 & 2008 & 2013 & Valor de $p * *$ \\
\hline \multicolumn{11}{|l|}{ Plano privado de saúde } \\
\hline Sem plano & 49,8 & 57,4 & 64,0 & 67,2 & 0,0001 & 34,7 & 40,1 & 41,3 & 45,2 & 0,0001 \\
\hline Com plano & 70,8 & 77,5 & 79,5 & 85,9 & 0,0001 & 67,0 & 77,1 & 73,1 & 74,3 & 0,0013 \\
\hline \multicolumn{11}{|l|}{ Sexo } \\
\hline Masculino & 43,0 & 49,5 & 55,2 & 61,6 & 0,0001 & 31,8 & 37,2 & 38,1 & 41,7 & 0,0001 \\
\hline Feminino & 66,7 & 74,3 & 79,4 & 79,6 & 0,0001 & 38,3 & 44,5 & 46,9 & 51,4 & 0,0001 \\
\hline \multicolumn{11}{|l|}{ Nível de instrução } \\
\hline Ensino Fundamental inicial incompleto ou menos & 52,3 & 58,7 & 64,0 & 65,7 & 0,0001 & 18,2 & 22,2 & 24,3 & 29,1 & 0,0001 \\
\hline Ensino Fundamental inicial & 53,0 & 59,7 & 65,7 & 67,8 & 0,0001 & 29,9 & 33,0 & 33,3 & 35,1 & 0,0001 \\
\hline Ensino Fundamental final & 52,6 & 59,2 & 64,1 & 68,2 & 0,0001 & 43,5 & 43,9 & 42,7 & 45,0 & 0,1010 \\
\hline Ensino Médio ou mais & 62,9 & 68,5 & 71,8 & 75,4 & 0,0001 & 56,3 & 58,8 & 55,5 & 57,7 & 0,0001 \\
\hline \multicolumn{11}{|l|}{ Faixa etária (anos) } \\
\hline $18-19$ & 44,0 & 50,9 & 56,3 & 62,4 & 0,0001 & 42,7 & 47,0 & 49,1 & 52,4 & 0,0001 \\
\hline $20-29$ & 50,5 & 57,4 & 62,9 & 68,7 & 0,0001 & 40,5 & 46,6 & 48,3 & 50,7 & 0,0001 \\
\hline $30-39$ & 55,3 & 62,5 & 67,9 & 70,3 & 0,0001 & 36,7 & 42,6 & 45,2 & 49,5 & 0,0001 \\
\hline $40-49$ & 59,3 & 66,7 & 71,0 & 72,8 & 0,0001 & 30,7 & 36,9 & 38,9 & 45,5 & 0,0001 \\
\hline $50-59$ & 64,9 & 71,2 & 75,3 & 77,1 & 0,0128 & 23,1 & 29,7 & 31,8 & 38,7 & 0,0001 \\
\hline
\end{tabular}

* Análises realizadas utilizando os pesos amostrais;

** Valores de p correspondentes ao teste de qui-quadrado com linearização de Taylor.

rem aumento do uso ao longo dos anos, apresentaram tendência de declínio a partir de 2003. Ainda assim, adultos com plano privado de saúde apresentaram chance maior de usar os serviços de saúde comparados àqueles sem plano em todos os anos analisados.

Um outro achado relevante é que há desigualdades educacionais no uso de serviços de saúde, em que os adultos com maior nível educacional consultaram mais o médico e o dentista. No entanto, em relação aos serviços odontológicos, tais desigualdades têm reduzido significativamente; em 1998, os adultos com Ensino Médio completo ou mais tinham 5,17 vezes mais chances de terem consultado o dentista no último ano, enquanto que, 15 anos após, esse valor caiu para pouco mais da metade. Essa tendência corrobora achados prévios 8,22. Já em relação ao uso dos serviços médicos, essas desigualdades educacionais aumentaram ao longo dos anos. Resultado diferente mostrando redução das desigualdades entre os mais ricos e os mais pobres na utilização de consultas médicas foi registrado entre as regiões Nordeste e Sudeste 23. É importante ressaltar que as variáveis de renda e educação utilizadas para medir desigualdades são diferentes, apesar de que a educação pode ser considerada como uma proxy para renda. É possível que os indivíduos de maior escolaridade estejam mais informados sobre sua saúde e utilizam mais os serviços médicos do que aqueles de menor escolaridade. E esse fato associado à ampliação da cobertura da ESF também pode ter facilitado e incentivado o acesso aos serviços de saúde por esses indivíduos. Considerando que todos os cidadãos podem usar o sistema público de saúde, essa desigualdade no uso pode ainda ter aumentado porque os indivíduos de maior escolaridade também têm acesso aos serviços médicos por meio do plano privado de saúde, uma vez que o maior percentual de pessoas com cobertura de planos privados de saúde encontra-se entre aqueles de maior renda e escolaridade.

As tendências no uso dos serviços médicos e odontológicos foram diferentes no período analisado. Apesar de ambos os serviços apresentarem tendência de aumento do uso entre os indivíduos com plano privado comparado àqueles sem cobertura de planos privados, a tendência no uso de serviços médicos no último ano aumentou no segundo ano, reduziu em 2008 e voltou a aumentar em 2013 


\section{Tabela 3}

Análise das tendências no uso de serviços médico e odontológico no último ano em relação à posse de plano privado de assistência médica e plano privado exclusivamente odontológico. Pesquisa Nacional por Amostra de Domicílios (PNAD 1998, 2003 e 2008) e Pesquisa Nacional de Saúde (PNS 2013), Brasil *.

\begin{tabular}{|c|c|c|c|c|}
\hline & \multicolumn{2}{|c|}{ Consulta médica } & \multicolumn{2}{|c|}{ Consulta odontológica } \\
\hline & OR & IC95\% & OR & IC95\% \\
\hline \multicolumn{5}{|l|}{ Sexo } \\
\hline Masculino & 1,00 & & 1,00 & \\
\hline Feminino & 2,56 & $2,47-2,66$ & 1,44 & $1,39-1,49$ \\
\hline \multicolumn{5}{|l|}{ Ano do Inquérito } \\
\hline 1998 & 1,00 & & 1,00 & \\
\hline 2003 & 1,37 & $1,30-1,44$ & 1,27 & $1,21-1,34$ \\
\hline 2008 & 1,78 & $1,61-1,95$ & 1,38 & $1,25-1,51$ \\
\hline 2013 & 1,94 & $1,67-2,24$ & 1,72 & $1,49-1,98$ \\
\hline \multicolumn{5}{|l|}{ Plano privado de saúde } \\
\hline Sem plano & 1,00 & & 1,00 & \\
\hline Com plano & 2,25 & $2,19-2,32$ & 2,48 & $2,21-2,78$ \\
\hline \multicolumn{5}{|l|}{ Ano-Plano privado de saúde ** } \\
\hline 2003-Plano & 1,03 & $0,99-1,07$ & 1,34 & $1,16-1,55$ \\
\hline 2008-Plano & 0,88 & $0,85-0,92$ & 1,14 & $1,01-1,30$ \\
\hline 2013-Plano & 1,23 & $1,13-1,33$ & 1,10 & $0,95-1,29$ \\
\hline \multicolumn{5}{|l|}{ Nível de instrução } \\
\hline Ensino Fundamental inicial incompleto ou menos & 1,00 & & 1,00 & \\
\hline Ensino Fundamental inicial & 1,07 & $1,04-1,10$ & 1,81 & $1,75-1,87$ \\
\hline Ensino Fundamental final & 1,08 & $1,04-1,12$ & 2,83 & $2,73-2,94$ \\
\hline Ensino Médio ou mais & 1,18 & $1,14-1,22$ & 5,17 & $5,01-5,34$ \\
\hline \multicolumn{5}{|l|}{ Nível de instrução-Ano (cont) ** } \\
\hline Ensino Fundamental inicial & 1,04 & $0,95-1,15$ & 0,71 & $0,65-0,79$ \\
\hline Ensino Fundamental final & 1,13 & $1,02-1,26$ & 0,64 & $0,57-0,71$ \\
\hline Ensino Médio ou mais & 1,19 & $1,09-1,30$ & 0,56 & $0,51-0,61$ \\
\hline \multicolumn{5}{|l|}{ Faixa etária (anos) } \\
\hline $18-19$ & 1,00 & & 1,00 & \\
\hline $20-29$ & 1,28 & $1,22-1,34$ & 0,90 & $0,86-0,95$ \\
\hline $30-39$ & 1,61 & $1,53-1,68$ & 0,82 & $0,78-0,86$ \\
\hline $40-49$ & 1,93 & $1,84-20,3$ & 0,67 & $0,64-0,71$ \\
\hline $50-59$ & 2,50 & $2,37-2,63$ & 0,56 & $0,53-0,59$ \\
\hline \multicolumn{5}{|l|}{ Faixa etária (anos)\#Ano (cont) ** } \\
\hline $20-29$ & 0,98 & $0,85-1,13$ & 0,93 & $0,81-1,07$ \\
\hline $30-39$ & 0,85 & $0,74-0,98$ & 1,03 & $0,90-1,18$ \\
\hline $40-49$ & 0,83 & $0,72-0,96$ & 1,19 & $1,04-1,37$ \\
\hline $50-59$ & 0,84 & $0,72-0,98$ & 1,22 & $1,05-1,41$ \\
\hline
\end{tabular}

* Ajustado por sexo, tendência na posse de plano privado, tendência na faixa etária e tendência no nível educacional com uso de pesos amostrais; ** A variável ano foi modelada como variável categórica para plano e como variável contínua para grau de instrução e faixa etária.

entre os indivíduos cobertos por planos privados. Estudo recente também registrou aumento do uso de consulta médica de 2008 para 2013 24. Resultados diferentes foram encontrados em outro estudo que registrou aumento da prevalência de consultas médicas de 2003 para 2008 entre os indivíduos com plano privado de saúde 23 , porém, este estudo considerou duas macrorregiões brasileiras nas análises e utilizou dados de consultas médicas no último ano realizadas por adultos e idosos. Essa diferença pode ser explicada pelo fato de não termos incluído idosos, uma vez que o percentual de 
cobertura por planos privados e o uso dos serviços médicos cresce com o aumento da idade. Outros estudos também encontraram maior uso de serviço médico entre indivíduos com posse de plano privado comparado àqueles sem plano, fortalecendo nossos achados 9,25,26,27 .

Indivíduos com cobertura de plano privado apresentaram maior uso de serviços odontológicos do que aqueles não cobertos em todos os anos analisados, os resultados são consistentes com estudos prévios 12,28,29. No entanto, apesar da tendência de uso ser maior entre os indivíduos cobertos por plano privado exclusivamente odontológico do que entre os sem plano, de 2003 a 2013, ocorreu diminuição no uso dos serviços pelos adultos com cobertura de plano privado apesar de ter aumentado o percentual de pessoas com plano privado exclusivamente odontológico nesse mesmo período. Ao contrário desse achado, estudo com adultos americanos mostrou que houve tendência recente de redução no uso de serviços odontológicos nos últimos anos, provavelmente, pela diminuição do percentual de cobertura por seguros de saúde 29 . Esse não é o caso brasileiro, uma vez que a posse de plano privado exclusivamente odontológico aumentou consideravelmente de 1998 para 2013. A redução no uso poderia ser explicada pela oferta de planos privados com cobertura limitada 30 que não atendem às necessidades do indivíduo. Apesar de que estudo realizado em outro país não encontrou diferença entre os níveis de cobertura e o uso dos serviços 12. A melhora nas condições de saúde bucal dos brasileiros ocorrida nos últimos anos provavelmente não explica essa redução no uso, pois, entre os adultos, os indicadores de necessidade são elevados 31 apesar da tendência de aumento de dentes em boas condições 32. Pesquisas são necessárias para explorar se o tipo de cobertura ou o copagamento influenciam o uso dos serviços de saúde no Brasil e se a posse de plano privado interfere nas condições de saúde da população.

Neste estudo, a posse de plano privado para assistência médica apresentou tendência de redução ao longo dos anos, ao contrário da posse de planos exclusivamente odontológicos que apresentou tendência de aumento. Poucos estudos têm avaliado a cobertura por planos privados odontológicos no sistema de saúde brasileiro. O número de beneficiários de planos privados odontológicos cresceu mais de $210 \%$ nos últimos anos 33 e, em nosso estudo, apesar da tendência de aumento na posse de plano, houve uma diminuição no uso dos serviços por indivíduos cobertos a partir de 2003. Em 2013, 5,2\% da população brasileira possuía plano exclusivo para atendimento odontológico, correspondendo a 10,3 milhões de pessoas ${ }^{19}$. Estudo recente mostrou que houve leve aumento na posse de plano privado de saúde na população brasileira 5, porém, esse não diferenciou o tipo de plano. É possível que a posse de plano privado de assistência médica esteja reduzindo entre a população brasileira e o aumento verificado seja explicado pelo grande aumento nos planos exclusivamente odontológicos. Outra questão a ser considerada é que, neste estudo, apenas a posse de plano privado entre adultos está sendo considerada.

Este estudo apresenta algumas limitações. Foi necessário fazer alguns ajustes metodológicos, estendendo as respostas dos titulares aos dependentes moradores do mesmo domicílio referente ao tipo de plano. Uma vez que apenas os titulares, nos anos de 1998 e 2003, responderam à questão relacionada à posse de plano exclusivamente odontológico. Além disso, não foi possível identificar o local de atendimento odontológico (público, privado ou por meio de planos de saúde) nesses mesmos anos. Com a identificação do local de atendimento, seria possível analisar se os indivíduos com plano privado estariam usando o serviço por meio desse ou estariam utilizando outros tipos de serviços. Outra limitação foi a impossibilidade de identificar, entre os planos de assistência médica, aqueles que oferecem cobertura odontológica no ano de 2013. Nesse ano, não foi perguntado aos entrevistados se o plano privado oferecia cobertura aos procedimentos odontológicos. Assim, optou-se por considerar somente os indivíduos com posse de plano privado exclusivamente odontológico, tendo um percentual de cobertura menor do que a real cobertura por plano odontológico. Entre as vantagens, este estudo inclui amostras representativas da população com abrangência nacional que possibilitam generalizar nossos resultados para toda a população e até para outros países com características semelhantes. Ainda, a periodicidade da coleta permite inferir tendências do uso de serviços, e as questões muito semelhantes nos quatro anos analisados mantêm a comparabilidade.

O presente estudo mostrou que há tendência de aumento do uso de serviços médicos e odontológicos entre indivíduos com e sem posse de plano privado, mas desigualdades no uso se perpetuam ao longo dos 15 anos analisados. Para o serviço médico, ocorreu variação no uso ao longo dos anos, mas, para o serviço odontológico, houve redução após o ano de 2003. O acompanhamento das tendências 
na posse de planos privados e no uso do serviço, bem como estudos para avaliar o estado de saúde e o tipo de serviço utilizado (público, pagamento direto do bolso ou por meio do plano) pelos indivíduos com plano privado de saúde são necessários para auxiliar o Estado na regulação dos planos e para evitar o aumento das iniquidades no acesso e no uso dos serviços entre os cidadãos.

\section{Colaboradores}

L. M. Pilotto e R. K. Celeste construíram o desenho do estudo, interpretaram os resultados, redigiram, leram e aprovaram o manuscrito final. R. K. Celeste realizou a análise dos dados.

\section{Agradecimentos}

À Coordenação de Aperfeiçoamento de Pessoal de Nível Superior (Capes) por ter concedido bolsa de estudos para L. M. Pilotto.

\section{Referências}

1. World Health Organization. Health systems financing: the path to universal coverage. Geneva: World Health Organization; 2010.

2. Barros AJD, Bastos JL, Dâmaso AH. Catastrophic spending on health care in Brazil: private health insurance does not seem to be the solution. Cad Saúde Pública 2011; 27 Suppl 2:S254-62.

3. Paim J, Travassos C, Almeida C, Bahia L, Macinko J. The Brazilian health system: history, advances, and challenges. Lancet 2011; 377:1778-97.

4. Organisation for Economic Co-operation and Development. Proposal for a taxonomy of health insurance. Paris: Organisation for Economic Co-operation and Development; 2004. (OECD Study on Private Health Insurance).

5. Viacava F, Bellido JG. Condições de saúde, acesso a serviços e fontes de pagamento, segundo inquéritos domiciliares. Ciênc Saúde Coletiva 2016; 21:351-70.

6. Garcia-Subirats I, Vargas I, Mogollón-Pérez AS, De Paepe P, Silva MRF, Unger JP, et al. Inequities in access to health care in different health systems: a study in municipalities of central Colombia and north-eastern Brazil. Int J Equity Health 2014; 13:10.

7. Lima-Costa MF, Matos DL, Camarano AA. Evolução das desigualdades sociais em saúde entre idosos e adultos brasileiros: um estudo baseado na Pesquisa Nacional por Amostra de Domicílios (PNAD 1998, 2003). Ciênc Saúde Coletiva 2006; 11:941-50.

8. Celeste RK, Nadanovsky P, Fritzell J. Trends in socioeconomic disparities in the utilization of dental care in Brazil and Sweden. Scand J Public Health 2011; 39:640-8.

9. Macinko J, Lima-Costa MF. Horizontal equity in health care utilization in Brazil, 1998-2008. Int J Equity Health 2012; 11:33.

10. Porto SM, Ugá MA, Moreira RS. Uma análise da utilização de serviços de saúde por sistema de financiamento: Brasil 1998-2008. Ciênc Saúde Coletiva 2011; 16:3795-806.

11. Manski RJ, Moeller JF, Chen H. Dental care coverage and use: modeling limitations and opportunities. Am J Public Health 2014; 104:2002-9.

12. Teusner D, Brennan D, Spencer A. Associations between level of private dental insurance cover and favourable dental visiting by household income. Aust Dent J 2015; 60:479-89. 
13. Moraes JR, Moreira JPL, Luiz RR. Associação entre o estado de saúde autorreferido de adultos e a área de localização do domicílio: uma análise de regressão logística ordinal usando a PNAD 2008. Ciênc Saúde Coletiva 2011; 16:3769-80.

14. Lima-Costa MF, Matos DL, Camargos VP, Macinko J. Tendências em dez anos das condições de saúde de idosos brasileiros: evidências da Pesquisa Nacional por Amostra de Domicílios (1998, 2003, 2008). Ciênc Saúde Coletiva 2011; 16:3689-96.

15. Bastos GA, Duca GF, Hallal PC, Santos IS. Utilização de serviços médicos no sistema público de saúde no Sul do Brasil. Rev Saúde Pública 2011; 45:475-84.

16. Instituto Brasileiro de Geografia e Estatística. Pesquisa Nacional por Amostra de Domicílios: análise de resultados, 1998. http://www. ibge.gov.br/home/estatistica/populacao/traba lhoerendimento/pnad98/saude/analise.shtm (acessado em 18/Ago/2013).

17. Instituto Brasileiro de Geografia e Estatística. Acesso e utilização de serviços de saúde. Rio de Janeiro: Instituto Brasileiro de Geografia e Estatística; 2003.

18. Instituto Brasileiro de Geografia e Estatística. Um panorama da saúde no Brasil: acesso e utilização dos serviços, condições de saúde e fatores de risco e proteção à saúde. Rio de Janeiro: Instituto Brasileiro de Geografia e Estatística; 2008.

19. Instituto Brasileiro de Geografia e Estatística. Pesquisa Nacional de Saúde 2013: acesso e utilização dos serviços de saúde, acidentes e violências. Brasil, grandes regiões e Unidades da Federação. Rio de Janeiro: Instituto Brasileiro de Geografia e Estatística; 2013.

20. Damacena GN, Szwarcwald CL, Malta DC, Souza Júnior PRB, Vieira MLFP, Pereira CA, et al. O processo de desenvolvimento da Pesquisa Nacional de Saúde no Brasil, 2013. Epidemiol Serv Saúde 2015; 24:197-206.

21. Szwarcwald CL, Malta DC, Pereira CA, Vieira ML, Conde WL, Souza Júnior PR, et al. Pesquisa Nacional de Saúde no Brasil: concepção e metodologia de aplicação. Ciênc Saúde Coletiva $2014 ; 19: 333-42$.
22. Luchi CA, Peres KG, Bastos JL, Peres MA. Inequalities in self-rated oral health in adults. Rev Saúde Pública 2013; 47:740-51.

23. Silva PSC, Boing AF, Peres KG. Redução das desigualdades no uso de consultas médicas no Brasil: análise das regiões Nordeste e Sudeste entre 2003 e 2008. Rev Bras Epidemiol 2015; 18:248-61.

24. Mullachery P, Silver D, Macinko J. Changes in health care inequity in Brazil between 2008 and 2013. Int J Equity Health 2016; 15:140.

25. Barros MB, Francisco PM, Zanchetta LM, Cesar CL. Tendências das desigualdades sociais e demográficas na prevalência de doenças crônicas no Brasil, PNAD: 2003-2008. Ciênc Saúde Coletiva 2011; 16:3755-68.

26. Jahangir E, Irazola V, Rubinstein A. Need, enabling, predisposing, and behavioral determinants of access to preventative care in Argentina: analysis of the national survey of risk factors. PLoS One 2012; 7:e45053.

27. Boccolini CS, Souza Júnior PR. Inequities in healthcare utilization: results of the Brazilian National Health Survey, 2013. Int J Equity Health 2016; 15:150.

28. Cornejo-Ovalle M, Paraje G, Vásquez-Lavin F, Pérez G, Palència L, Borrell C. Changes in socioeconomic inequalities in the use of dental care following major healthcare reform in Chile, 2004-2009. Int J Environ Res Public Health 2015; 12:2823-36.

29. Wall TP, Vujicic M, Nasseh K. Recent trends in the utilization of dental care in the Unites States. J Dent Educ 2012; 76:1020-7.

30. Finkler M, Castro RG, Mello ALSF, Caetano JC. A relação público-privada na odontologia brasileira. Revista de Saúde Pública de Florianópolis 2009; 2:91-112.

31. Pucca Jr. GA, Gabriel M, Araújo ME, Almeida FC. Ten years of a National Oral Health Policy in Brazil: innovation, boldness, and numerous challenges. J Dent Res 2015; 94:1333-7.

32. Celeste RK, Nadanovsky P, Fritzell J. Trends in socioeconomic disparities in oral health in Brazil and Sweden. Community Dent Oral Epidemiol 2011; 39:204-12.

33. Pietrobon L, Silva CM, Batista LRV, Caetano JC. Planos de assistência à saúde: interfaces entre o público e o privado no setor odontológico. Ciênc Saúde Coletiva 2008; 13:1589-99. 


\section{Abstract}

The public-private mix in the Brazilian health system favors double coverage of health services for individuals with private health plans and may aggravate inequities in the use of services. The aim of this study was to describe trends in the use of medical and dental services and associations with schooling and private health coverage. Data were obtained from a national household survey with representative samples in the years 1998, 2003, 2008, and 2013. The study described trends in the use of health services by adults, adjusted by private health coverage, years of schooling, sex, and age. There was an upward trend in the use of health services in adults without a private plan and among adults with a private plan the trend in use varied in a non-linear way. The medical service presented alternation in use over the years and the dental service showed a tendency to decline after 2003. It is necessary to monitor trends in private health coverage and the use of health services to assist government in regulating private plans and avoid increasing inequities among citizens in access to and use of health services.

Health Services; Universal Coverage; Health Insurance

\section{Resumen}

El mix público-privado del sistema de salud brasileño favorece la cobertura duplicada a los servicios de salud para individuos que posean un plan privado de salud, y puede aumentar las inequidades en el uso de los servicios. El objetivo de este estudio es describir las tendencias en el uso de los servicios de salud médicos y odontológicos, y su relación con el nivel educacional y la tenencia de un plan privado de salud. Los datos se obtuvieron de encuestas domiciliarias nacionales, con muestras representativas de los años de 1998, 2003, 2008 y 2013. Se describieron las tendencias en el uso de servicios de salud por parte de adultos, ajustadas por la tenencia de un plan privado de salud, nivel de educación, sexo y edad. Existe una tendencia de aumento en el uso de los servicios de salud en adultos sin plan privado y, entre los adultos con plan privado, la tendencia en el uso varió de forma no lineal. El servicio médico presentó alternancia en el uso a lo largo de los años y el servicio odontológico presentó tendencia de declinación después del año 2003 Acompañar las tendencias en la obtención de planes privados de salud y en el uso de los servicios de salud es necesario para auxiliar al Estado en la regulación de los planes privados, y así evitar el aumento de las inequidades en el acceso y uso de los servicios de salud entre los ciudadanos.

Servicios de Salud; Cobertura Universal; Seguro de Salud
Recebido em 27/Mar/2017

Versão final reapresentada em 09/Jun/2017

Aprovado em 07/Ago/2017 